\title{
Concatenated Raptor Codes in NAND Flash Memory
}

\author{
Geunyeong Yu, Member, IEEE, and Jaekyun Moon, Fellow, IEEE
}

\begin{abstract}
Two concatenated coding schemes based on fixedrate Raptor codes are proposed for error control in NAND flash memory. One is geared for off-line recovery of uncorrectable pages and the other is designed for page error correction during the normal read mode. Both proposed coding strategies assume harddecision decoding of the inner code with inner decoding failure generating erasure symbols for the outer Raptor code. Raptor codes allow low-complexity decoding of very long codewords while providing capacity-approaching performance for erasure channels. For the off-line page recovery scheme, one whole NAND block forms a Raptor codeword with each inner codeword typically made up of several Raptor symbols. An efficient lookup-table strategy is devised for Raptor encoding and decoding which avoids using large buffers in the controller despite the substantial size of the Raptor code employed. The potential performance benefit of the proposed scheme is evaluated in terms of the probability of block recovery conditioned on the presence of uncorrectable pages. In the suggested page-error-correction strategy, on the other hand, a hard-decision-iterating product code is used as the inner code. The specific product code employed in this work is based on row-column concatenation with multiple intersecting bits to allow the use of longer component codes. In this setting the collection of bits captured within each intersection of the row-column codes acts as the Raptor symbol(s), and the intersections of failed row codes and column codes are declared as erasures. The error rate analysis indicates that the proposed concatenation provides a considerable performance boost relative to the existing error correcting system based on long BoseChauduri-Hocquenghem (BCH) codes.
\end{abstract}

Index Terms-Raptor codes, error-correction-code, flash memory, BCH codes, product codes

\section{INTRODUCTION}

$\mathbf{N}$ AND flash memories have become immensely popular due to lower power consumption, stronger shock resistance and higher data throughputs relative to hard-disk drives. Recently, high-capacity NAND flash memories have been increasingly deployed in USB drives, solid state drives (SSDs) and all forms of mobile devices. However, the high cost-perbit remains the main weakness of NAND flash memories. As the demand for cost reduction continues, multi-level cells (MLCs) which contain more than two bits per memory cell are also being widely employed. The MLC-based NAND flash can improve storage density, but the increased sensitivity to noise presents a critical issue. Aggressive technology scaling has also contributed significantly to the bit-cost reduction

Copyright (c) 2013 IEEE. Personal use of this material is permitted. However, permission to use this material for any other purposes must be obtained from the IEEE by sending a request to pubs-permissions@ieee.org.

Geunyeong Yu and Jaekyun Moon are with the Department of of Electrical Engineering, Korea Advanced Institute of Science and Technology (KAIST) Daejeon 305-701, Republic Korea (e-mail: tp10dal@stein.kaist.ac.kr, jmoon@kaist.edu). This work was supported by the National Research Foundation of Korea under grant no. 2013-13057165, the Ministry of Knowledge Economy of Korea under grant no. 10035202, and SK hynix. Geunyeong Yu has been partially supported by Samsung Electronics. of NAND flash memory. However, technology scaling also results in the reduced noise margin and increased cell-to-cell interference.

To get around these issues, combinations of hardware and software techniques are typically employed. The controller of NAND flash systems tries to distribute the program/erase cycles over the entire set of data blocks to prolong the lifetime of flash memories, a scheme called wear leveling [1]. A bad block management technique is employed to keep track of bad blocks of which uncorrectable page errors have been encountered, preventing writing of data to the known bad blocks. Another essential element of the modern storage system controller is the error-correcting code (ECC) used to ensure data reliability.

The trend in ECC is to employ increasingly strong codes with significantly improved error correction capability. In fact, the raw channel bit error rate (BER) before error correction is now allowed to reach $10^{-3}$ and higher. While long $\mathrm{BCH}$ codes are still very popular, the low-density parity-check (LDPC) [2]-[5] and turbo codes have emerged as promising candidates as well [6]. But both LDPC codes and turbo codes require soft read signals off the NAND chips to realize full performance potential, and given the way all cells in a common word line is read simultaneously via comparison against a uniform threshold level, generating soft read values for the NAND memory cells necessitates multiple reads with varying sensing levels [4]. Multiple reads directly translate to increased system latency and thus present difficulty in delay-sensitive applications.

While strong error protection is also possible using very long $\mathrm{BCH}$ codes, decoding complexity increases rapidly with the error correction capability and the codeword length. One way of utilizing the existing binary $\mathrm{BCH}$ codes to strengthen page error protection is to apply product concatenation of $\mathrm{BCH}$ codes along row and column directions of a two-dimensional buffer. In particular, generalized product concatenation of $\mathrm{BCH}$ codes can allow each pair of row and column codes to intersect at multiple bits rather than at a single bit as in the traditional product code based on binary component codes [7]. This block-wise product code (BW-PC) allows the use of stronger component codes for a given overall codeword size while compromising the ability of locating specific erroneous bit positions of the usual bit-wise product concatenation.

In this paper, we focus on concatenated codes based on Raptor codes, efficient erasure-correcting codes originally presented as a type of fountain codes ideal for multi-casting or broad-casting in network communications. Fountain codes, including Raptor codes, used in the network communication setting are often described as "rateless" in the sense that the number of encoder packets is not fixed before transmission. The encoder in these codes continues to generate coded 
packets (or symbols) as (pseudo) random linear combinations of source symbols and transmits them until all the intended receivers have recovered all the original source symbols. It is known that Raptor codes are asymptotically capacityapproaching in erasure channels whether the erasure probability are available at the transmitter or not [8]. Practical and theoretic aspects of Raptor codes have been the subject of ongoing studies [9]-[15].

We shall consider here the application of a specific Raptor code known as the R10 code [15]-[17] in a fixed-rate setting. This code exhibits optimal error rate performance as a random fountain code which has a decoding error probability of $2^{-\left(N^{\prime}-K\right)}$ where $N^{\prime}$ is the number of correctly received symbols and $K$ is the number of source symbols. The R10 code has two important desirable characteristics: it is a systematic code and efficient decoding is possible based on what is called inactivation decoding. Inactivation decoding employs belief propagation (BP) decoding as much as possible while also attempting to solve a linear system of equations when necessary, in an effort to realize efficient overall decoding without compromising the performance of near-maximumlikelihood decoding. Because of its superior performance and linear-time encoding/decoding feature, the Raptor code has been adopted by the 3rd Generation Group Partnership Project (3GPP) to be used in multimedia broadcast/multicast services (MBMS) for forward error correction [17] and digital video broadcast-handheld (DVB-H) systems [18]. The application of Raptor codes to binary-input memoryless symmetric channels in a fixed-rate setting has also been studied in [19], [20].

In this paper, we specifically consider 1 ) off-line recovery of contaminated pages and 2) real-time page error correction, both based on concatenation of BCH-Raptor codes. In current NAND flash memory systems, reading and writing are done on a page by page basis. Each page contains several kilo bytes while each NAND block is made up of 128 to 256 pages. In our proposed off-line page recovery mode, each page is divided into several words. A large number of such words from all pages within the given NAND block forms a Raptor codeword with each word corresponding to one or more Raptor symbols. In the proposed setting, several Raptor symbols also form an inner BCH codeword. In this setting, the Raptor code attempt to clean up erasures that may arise as a result of the inner decoding failure. With typical code system parameters chosen, a significant number of uncorrectable pages within a block can be recovered with a very small extra coding overhead.

In some of current advanced NAND flash memory systems, extra protection for the contaminated pages that cannot be corrected by the deployed ECC (due to e.g. physical damages or wear in the block of data written overtime) is done via the redundant arrays of inexpensive disks (RAID) architecture based on the distribution of data and its parity across several NAND chips [21], [22]. However, RAID systems require a high level of redundancy in storing parity. In contrast, a Raptor code applied to the whole NAND block provides protection against failed pages with a very small extra coding overhead while allowing for efficient encoding and decoding. The main challenge in our use of the Raptor code in the page recovery mode is that the codeword of the Raptor code corresponds to the entire NAND block, which is in the order of mega bytes in size. While this size is way too large for a practical controller to handle, our proposed scheme based on tablelookup strategies allows encoding and decoding using only a small amount of buffers. Compared to the RAID system, our scheme does not allow real-time extra page protection; the low-complexity and low-overhead features come at the cost of a slower processing speed.

On the other hand, in our proposed page-error-correction mode during normal page reads, a Raptor code is concatenated with an inner BW-PC based on relatively small $\mathrm{BCH}$ component codes. Iterative hard-decision-decoding is utilized for the inner code. In this setting the collection of bits captured within each intersection of the row-column codes acts as one or more Raptor symbols, and the intersections of failed row codes and failed column codes after BW-PC decoding are declared as erasures for the outer Raptor code. Here, each page basically corresponds to a single Raptor codeword. Our error rate analysis indicates that the proposed concatenation provides a considerable performance boost relative to the existing BCH-based coding schemes.

Note that while the Reed-Solomon (RS) codes are also a clear option as the outer code, decoding complexity grows quickly with the increasing block size. The RS codes are deemed a less attractive solution for the problems at hand.

The term "block" in the block-wise product code (BW-PC) of [7] is not to be confused with the term "block" in the typical description of the NAND block structure. To avoid ambiguity, the block of bits captured in the intersections of row and column codewords will also be referred to as symbol in this paper, although the context will usually make the intended meanings clear.

The paper is organized as follows. In Section $\amalg$ we briefly review Raptor codes. In Section [II] we describe the Raptorcode-based off-line page recovery scheme. The detailed encoding and decoding algorithms of the Raptor code used in the page recovery mode are described and the error rate performance is evaluated. In Section IV the second concatenated Raptor code geared for real-time page error correction is discussed. It is shown that the channel is effectively converted from a binary symmetric channel (BSC) to a symbol-erasure channel via the use of the inner BW-PC. The error rate performance for the BW-PC and Raptor decoding is also evaluated in this section. Finally, we conclude our work in Section V.

\section{BACKGROUND}

\section{A. Fountain Codes}

Fountain codes are a type of erasure codes first proposed for multi-cast network communications [9], [10]. Given a source data consisting of $K$ symbols (frequently referred to as packets in the fountain code literature), the encoder of a fountain code produces a stream of output symbols, each of which is generated independently as some linear combination of the source symbols with the linear mapping chosen anew each time in a pseudo-random fashion. It is possible to recover 
the original $K$ symbols from any set of $N=K(1+\epsilon)$ received symbols with high probability (at least $1-1 / K^{c}$ ), where $c$ is a constant. The number of encoded packets is not fixed beforehand and transmission typically continues until all receivers (in the multi-cast mode) recover all source symbols. An encoder potentially generating an endless stream of packets in all directions until all receivers are satisfied conjures up the image of a fountain, hence the name fountain codes. Also, this class of codes is often referred to as rateless since the number of encoded packets is not fixed before the transmission, which in the context of traditional error control coding is to say that the code rate is not predetermined. At the transmitter and the receiver resides the same generator matrix for the linear mappings of the source symbols to the transmitted packets. When the coded packets or symbols are transmitted, the receiver throws out the erased symbols and reduces the generator matrix accordingly before decoding the original source symbols.

From the complexity view point, to encode each symbol requires $K / 2$ symbol additions; since a total of $N \approx K$ coded symbols are transmitted, encoding complexity can be described as $K(K / 2)$. A conceptually simple decoding strategy would be to perform a matrix inversion and, using Gaussian elimination, this will cost about $K^{3}$ symbol additions.

Luby transform (LT) codes represent the first practical realization of fountain codes [23]. LT codes use the low-density generator matrix (LDGM) and message-passing decoding to avoid direct matrix inversion. With a careful choice of the node degree distributions in the code's factor graph, it has been shown that the LT decoder can recover all $K$ source symbols with small coding redundancy. However, a significant drawback remains: namely, the average node degree is $O(K \ln K)$, which does not quite allow linear-time encoding/decoding.

Raptor codes [8], an extension of LT codes, are designed to achieve linear-time encoding/decoding. Raptor codes employ an outer code concatenated with a weakened LT code which has a significantly low average node degree. An LT code with a lower average node degree is normally problematic since there exists a significant probability that a source symbol may not be connected to clean received symbols in the factor graph, a situation that leads to decoding failure. But in Raptor codes, these unconnected source symbols can be corrected by an outer code with a very small coding redundancy. Overall, Raptor codes can achieve nearly linear-time encoding/decoding and has been proved to be asymptotically (as $K$ tends to infinity) capacity-achieving on a binary erasure channel (BEC) without any channel information at the transmitter or at the receiver [8].

\section{B. Raptor Encoding and Decoding}

A fountain code can also be viewed as a regular linear block code which can be represented by a generator matrix. This view facilitates exposition of the encoding and decoding processes of Raptor codes. We specifically describe encoding and decoding of the R10 code, which is a systematic code and allows simple evaluation of the error rate performance for low erasure-rate channels. The description in this subsection is basically a quick overview of the algorithms given in [15], [16].

\section{Encoding process}

There are basically two steps in the encoding process. The first step is to generate some $L$-intermediate symbols from the source symbols. Let us assume that the code length is $N$. Let $\mathbf{m}$ denote the vector of $L$ intermediate symbols and let $\mathbf{t}=\left[\begin{array}{ll}\mathbf{z}^{T} & \mathbf{s}^{T}\end{array}\right]^{T}$ where $\mathbf{z}$ is a vector of $(S+H)$ zero symbols and $\mathbf{s}$ is a vector of $K$ source symbols with ()$^{T}$ indicating transpose. Then $\mathbf{m}$ and $\mathbf{t}$ are related by $\mathbf{A}_{\text {pre }} \mathbf{m}=\mathbf{t}$, where $\mathbf{A}_{\text {pre }}$ is an $L \times L$ matrix over $G F(2)$ and $L=K+S+H$. Here, the first $(S+H)$ rows of $\mathbf{A}_{\text {pre }}$ describe the relationship among the intermediate symbols and the last $K$ rows contain connection information between the intermediate symbols and source symbols, consistent with the designed degree distribution. Hence, the intermediate vector $\mathbf{m}$ can be produced as:

$$
\mathbf{m}=\mathbf{A}_{\text {pre }}^{-1} \mathbf{t} .
$$

Once the intermediate symbols are determined, then in the second step $N-K$ redundant symbols are encoded by the LT relationships depicted by $\mathbf{G}_{L T}$, which is an $M \times L$ binary matrix with $M=N-K$. The LT generator matrix can produce any number of parity symbols $\mathbf{r}$ according to

$$
\mathbf{r}=\mathbf{G}_{L T} \mathbf{m},
$$

and the coded vector $\mathbf{c}$ can be written as

$$
\mathbf{c}=\mathbf{A m}=\left[\begin{array}{lll}
\mathbf{z}^{T} & \mathbf{s}^{T} & \mathbf{r}^{T}
\end{array}\right]^{T}
$$

where

$$
\mathbf{A}=\left[\begin{array}{l}
\mathbf{A}_{\text {pre }} \\
\mathbf{G}_{L T}
\end{array}\right] .
$$

Here, we note that $\mathbf{A}$ is known to both the encoder and the decoder and the value of $M$ is selected to be sufficiently large to compensate for possible loss of encoded symbols in the channel.

\section{Decoding process}

After the coded vector (excluding the first $S+H$ zeros symbols) is transmitted through the channel, decoding also consists of two basic steps - the first step for finding the intermediate symbols and the second step for recovering the erased symbols using the original generator matrix $\mathbf{A}$. Let us assume that $\mathbf{c}^{\prime}$ is the vector of successfully received symbols followed by a length- $(S+H)$ all-zero vector, $N^{\prime}$ is its length and $\mathbf{A}^{\prime}$ is the reduced generator matrix obtained from $\mathbf{A}$ by eliminating the rows corresponding to the erased symbols. Therefore, the success of decoding depends on whether $\mathbf{A}^{\prime} \mathbf{m}=\mathbf{c}^{\prime}$ can be solved or not. If the intermediate symbol vector $\mathbf{m}$ can be obtained via matrix inversion, then in the second step the erased symbols of $\mathbf{c}$ can be reproduced using $\mathbf{A}$ and $\mathbf{m}$.

\section{Matrix Inversion and Inactivation Decoding}

Decoding and encoding of Raptor code amount to solving the equations $\mathbf{A}^{\prime} \mathbf{m}=\mathbf{c}^{\prime}$ and $\mathbf{A m}=\mathbf{t}$, respectively, as described above. To obtain $\mathbf{m}$ from $\mathbf{c}^{\prime}$, Gaussian elimination 
(GE) can be performed. Efficient decoding algorithms have been suggested for this in [17], [15] and an improved implementation of maximum-likelihood (ML) decoding algorithm has also been presented in [12] to solve the same problem. These algorithms are different mainly in their ordering of the rows for Gaussian elimination, but they all give the same result. The objective is to turn $\mathbf{A}^{\prime}$ into an identity matrix by row exchanges, column exchanges and row additions.

The decoding algorithm can also be described by the belief propagation (BP) decoding [2], [24], [25]. In the case of the erasure channel, the BP algorithm is best described in terms of the "decoding graph" corresponding to the relationship between the received coded symbols and the intermediate symbols. This is a bipartite graph between the $L$ intermediate symbols and the $N^{\prime}$ received encoded symbols. Note that the BP algorithm for general LT or Raptor codes can be described [26] as: 1) finding a degree-1 encoded symbol, 2) delivering the value of the chosen encoded symbol to the connected source symbol (or intermediate symbol in the case of a Raptor code), 3) exclusive-ORing the given values to all the encoded symbols adjacent to the selected source (or intermediate) symbol and 4) eliminating all edges emanating from the source (or intermediate) symbol. The steps 1) though 4) are performed iteratively until there remain no unrecovered source (or intermediate) symbols or there is no degree-1 encoded symbol left in the graph. In comparison, in the decoding algorithm geared to the R10 code, the decoding process is effectively allowed to continue even if there is no degree- 1 encoded symbol in the graph via a special procedure called inactivation decoding [15]. This can be envisioned as allowing the BP algorithm to continue by removing (or inactivating) certain edges and nodes even after the BP algorithm gets stuck in the conventional graph. Once BP decoding halts, a simple linear system of equations arises according to the inactivated notes and edges; if there exists a solution, then the whole intermediate symbols can be recovered. It can be shown that decoding is possible overall whenever the reduced matrix $\mathbf{A}^{\prime}$ is full rank. Assuming a matrix size of $q$, solving it requires exclusive-OR operations in the order of $q^{2}$. Thus, if $q$ is in the order of $\sqrt{K}$, then the computational cost can be said to be linear in $K$. The R10 code of [15] is actually designed to satisfy this condition, requiring $O(K)$ symbol additions for overall decoding.

\section{RAPTOR CODE IN BLOCK RECOVERY}

In this section, we introduce a Raptor-code-based concatenation scheme where each codeword of the inner code encompasses several Raptor symbols. In this setting the entire NAND block of flash memory cells become a single Raptor codeword. The channel model of NAND flash is assumed to be a BSC in this paper since all decoding is based on harddecision inputs. The overall code construction for the proposed scheme along with detailed encoding and decoding algorithms are described, and the error rate performance is evaluated.

The simplest way to convert a BSC to a packet-erasure channel is to use an existing $(n, k)$ inner error detection or correction code, where $n$ is the codeword length and $k$ is

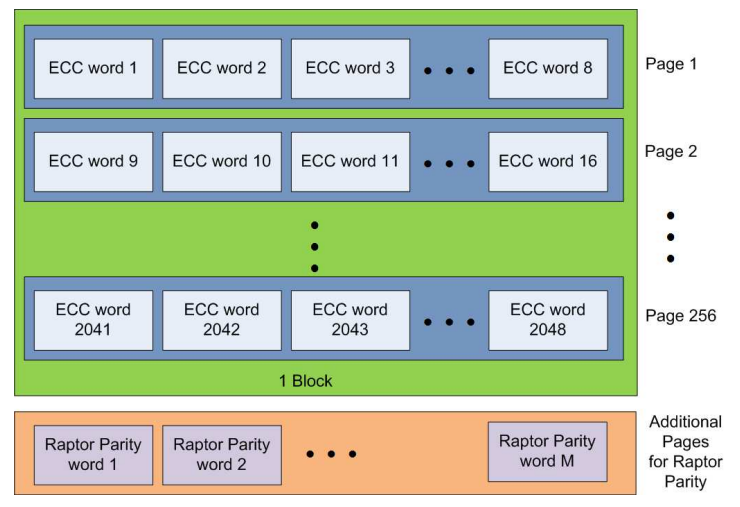

Fig. 1. Raptor Codes in NAND flash memory for the block recovery $\left(N_{s}=1\right.$, $p=256, w=8$ )

the information word length. Any linear block codes such as the LDPC, BCH code and Reed-Solomon (RS) codes can be used as the inner code. While the error detection code in general allows a high overall code rate, the erasure rate of the converted channel can be excessively high. In our investigation, inner ECCs with a reasonably high rate have proven to be a better choice. Our discussion in this paper will focus on the use of inner ECCs.

We will specifically use the $\mathrm{BCH}$ codes as inner codes. We assume that one NAND block consists of $p$ pages and one page contains $w$ inner codewords. We also assume that each codeword of the inner $\mathrm{BCH}$ code exactly contains $N_{s}$ source symbols of the Raptor code. Fig. 11 shows an example of the Raptor code applied to page recovery when $N_{s}=1, p=256$ and $w=8$. Let $K$ be the number of inner ECC codewords in one block (i.e., $K=w p$ ), and the total size of the Raptor parity symbols be equal to that of $N-K$ inner codewords. The Raptor codeword contains $n N$ coded bits corresponding to $k K$ information bits, and there exist $N_{s} N$ Raptor symbols consisting of $N_{s} K$ source symbols and $N_{s}(N-K)$ parity symbols. In the overall decoding process, $N_{s}$ symbols are declared as erasures whenever the inner decoder fails, and the Raptor decoder then attempts to recover the erased source symbols.

\section{A. Encoding and Decoding Algorithm}

As mentioned earlier, the NAND block size is in the order of mega bytes, and direct encoding and decoding of a Raptor code of this size are not feasible due to the required memory buffer size. To this end, we devise an efficient algorithm which uses only small buffers for the encoding and decoding processes. The key idea is that the lookup table of each parity symbol can be pre-calculated in the form of the position vector which contains the addresses of the required source symbols to generate the given parity symbol. Likewise in the proposed decoding process, the look-up tables for the erased symbols declared after inner decoding also are pre-calculated in the forms of the position vectors of the required received symbols.

In the encoding process, as the input data stream gets to the 
NAND block 1 the required source symbols are exclusive-ORed to calculate each parity symbol according to the pre-calculated look-up table. In decoding, if the recovery mode is invoked by the corrupted words or pages, only the required symbols are read to recover the erased symbols. This is possible because the parity symbols and the erased symbols are simple modulo sums of known sets of the source symbols and the encoded symbols, respectively. We note that the look-up tables of parity symbols are pre-calculated only once and used repeatedly, but the look-up tables of erased symbols need be calculated according to the positions of the erasures.

The look-up table of the erased symbols can be obtained in the proposed decoding process as follows. Decoding of a Raptor code basically consists of solving

$$
\mathbf{A}^{\prime} \mathbf{m}=\mathbf{c}^{\prime}
$$

for $\mathbf{m}$ and then regenerating the erased symbol using $\mathbf{m}$ and A as described in the subsection $\amalg-B$ To solve $[5$, Gaussian elimination should be performed which turns $\mathbf{A}^{\prime}$ into the form of an identity matrix by row exchanges, column exchanges and row additions. Whenever two rows of $\mathbf{A}^{\prime}$ are exchanged, the corresponding symbols of $\mathbf{c}^{\prime}$ are also exchanged, and when two columns of $\mathbf{A}^{\prime}$ are exchanged, the corresponding symbols of $\mathbf{m}$ are also exchanged. When the $i$-th row of $\mathbf{A}^{\prime}$ is added to the $j$-th row, the $i$-th symbol of $\mathbf{c}^{\prime}$ is also added to the $j$-th symbol. This process can be traced by multiplying the identity matrix to right side of (5):

$$
\mathbf{A}^{\prime} \mathbf{m}=\mathbf{I}_{N^{\prime}} \mathbf{c}^{\prime}
$$

and then transforming (6), through Gaussian elimination, to:

$$
\mathbf{I}_{L} \mathbf{m}^{\prime}=\mathbf{P c}^{\prime}
$$

where $\mathbf{m}^{\prime}$ is the reordered intermediate symbol vector. Then, $m_{i}^{\prime}$, the $i$-th symbol of $\mathbf{m}^{\prime}$ can be written as $\mathbf{p}_{\mathbf{i}} \mathbf{c}^{\prime}$, where $\mathbf{p}_{\mathbf{i}}$ is a row vector corresponding to the $i$-th row of $\mathbf{P}$. The erasure symbols can finally be reconstructed from $\mathbf{c}=\mathbf{A m}$.

Let the look-up table for the $k$-th parity symbol be $\mathbf{b}_{k}^{p}$ with the $l$-th component $b_{k, l}^{p}$ set to ' 1 ' when the $l$-th source symbol is necessary to generate the given parity symbol, and to ' 0 ' otherwise. Likewise, let the look-up table for the erased symbol corresponding to the $j$-th coded symbol be the binary vector $\mathbf{b}_{j}^{e}$ with the $i$-th component $b_{j, i}^{e}$ set to ' 1 ' when the $i$-th received symbol is necessary to recover the erased symbol, and to ' 0 ' otherwise.

Let us consider a specific example where the equality $\mathbf{A m}=\mathbf{c}$ is given by:

$$
\left[\begin{array}{llll}
0 & 1 & 0 & 1 \\
1 & 0 & 1 & 1 \\
0 & 1 & 0 & 0 \\
0 & 0 & 1 & 1 \\
1 & 0 & 0 & 1
\end{array}\right]\left[\begin{array}{l}
m_{1} \\
m_{2} \\
m_{3} \\
m_{4}
\end{array}\right]=\left[\begin{array}{l}
s_{1} \\
s_{2} \\
s_{3} \\
s_{4} \\
r_{1}
\end{array}\right]
$$

where the last source symbol $r_{1}$ represents the sole parity symbol. Assume that $s_{4}$ is erased in the channel. Then, after

\footnotetext{
${ }^{1}$ Notice that although our exposition encourages visualization of coding on the entire physical NAND block, our scheme actually employs logical blocks so that codewords may not necessarily come from a common physical NAND block.
}

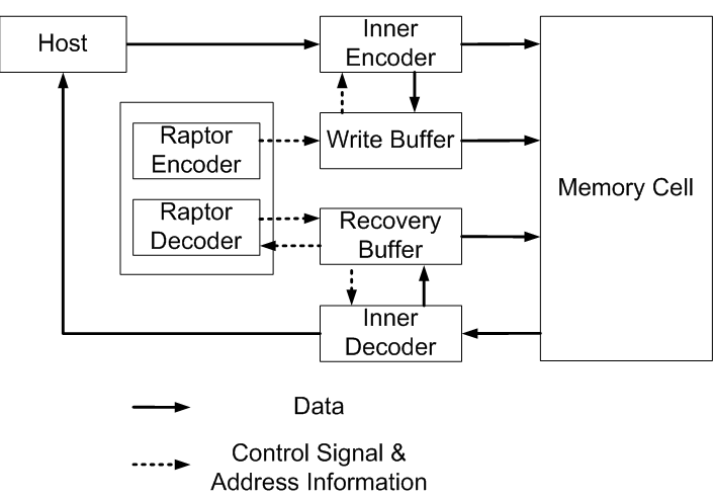

Fig. 2. Overall System Diagram for the Raptor encoder and decoder.

eliminating $s_{4}$, the equation $\mathbf{A}^{\prime} \mathbf{m}=\mathbf{c}^{\prime}$ becomes (8) reduces to

$$
\left[\begin{array}{llll}
0 & 1 & 0 & 1 \\
1 & 0 & 1 & 1 \\
0 & 1 & 0 & 0 \\
1 & 0 & 0 & 1
\end{array}\right]\left[\begin{array}{l}
m_{1} \\
m_{2} \\
m_{3} \\
m_{4}
\end{array}\right]=\left[\begin{array}{l}
s_{1} \\
s_{2} \\
s_{3} \\
r_{1}
\end{array}\right]
$$

To obtain the look-up table for the erased source symbol $s_{4}$, Gaussian elimination is executed which turns $\mathbf{A}^{\prime}$ in $(9)$ into an identity matrix by row/column exchanges as well as row additions. The transformed equality $\mathbf{I}_{L} \mathbf{m}^{\prime}=\mathbf{P} \mathbf{c}^{\prime}$ is now

$$
\left[\begin{array}{llll}
1 & 0 & 0 & 0 \\
0 & 1 & 0 & 0 \\
0 & 0 & 1 & 0 \\
0 & 0 & 0 & 1
\end{array}\right]\left[\begin{array}{l}
m_{2} \\
m_{4} \\
m_{1} \\
m_{3}
\end{array}\right]=\left[\begin{array}{llll}
0 & 0 & 1 & 0 \\
1 & 0 & 1 & 0 \\
1 & 0 & 1 & 1 \\
1 & 1 & 1 & 1
\end{array}\right]\left[\begin{array}{c}
s_{1} \\
s_{2} \\
s_{3} \\
r_{1}
\end{array}\right] .
$$

The intermediate symbols can be expressed as $m_{2}=\mathbf{p}_{\mathbf{1}} \mathbf{c}^{\prime}$, $m_{4}=\mathbf{p}_{2} \mathbf{c}^{\prime}$ and so on. To recover $s_{4}$, we go back to 8 to find that $s_{4}$ is sum of $m_{3}$ and $m_{4}$. Hence, $s_{4}$ is now reconstructed as $\left(\mathbf{p}_{\mathbf{2}}+\mathbf{p}_{\mathbf{4}}\right) \mathbf{c}^{\prime}$ and the corresponding look-up table is [01001], indicating that the second and fifth encoded symbols are used to recover the given symbol.

Note that the off-line recovery mode is invoked only in the presence of uncorrectable inner codewords. The overall system diagram of the Raptor encoder and decoder is shown in Fig. 2. For the case where each word protected by the inner code within a block is a single Raptor symbol (i.e. $N_{s}=1$ ), the overall encoding and decoding steps are summarized as follows:

\section{Encoding process}

1. Before the data is written into the NAND flash memory cells, the Raptor encoder generates the look-up tables $\mathbf{b}_{k}^{p}$ for $k=1,2, \ldots, N-K$ ) and then stores them, in the write buffer.

2. While the data is being written into the memory cells, for the $l$-th incoming word $W_{l}$,

a) the write buffer checks if $b_{k, l}^{p}$ is ' 1 ' for all $k$.

b) if true, the parity symbol $P_{k}$ (initially set to all zeros)

is updated after being exclusive-ORed with $W_{l}$.

c) repeat steps a) and b) for all $l$ values. 
3. When the $j$-th word of a given NAND block is updated, the write buffer updates the appropriate parity words by exclusive-ORing them with the new word.

\section{Decoding process}

1. While reading the data from the memory cells, if uncorrectable errors occur which cannot be recovered by the inner codes, the recovery buffer marks them as the erasure words, and saves their positions. The position information for the erasure words are delivered to the Raptor decoder.

2. For each erasure word $E_{j}$ in position $j$, the look-up table $\mathbf{b}_{j}^{e}$ is generated and delivered to the recovery buffer.

3 . When the off-line recovery mode is invoked, necessary words are read from the memory cells and cleaned up using the inner decoder. For the resulting $i$-th word $W_{i}$, a) the recovery buffer checks if $b_{j, i}^{e}$ is ' 1 ' for all $j$.

b) if true, $E_{j}$ is exclusive-ORed with $W_{i}$.

4. After all erased symbols $E_{j}$ are recovered, they are written to new logical memory cell locations.

The proposed recovery scheme based on Raptor codes can recover the corrupted words which cannot be recovered by the inner codes. By utilizing the pre-calculated look-up tables, efficient encoding and decoding are possible without directly handling a block of data, the size of which is in the order of mega bytes, making direct encoding and decoding practically infeasible. Here, we should point out that the proposed scheme requires re-writable memory for maintaining and updating parity symbols as well as extra memory to store the look-up tables for encoding and decoding. But in our proposed system setting, the parity portion in a block represents a very small percentage of the block and the size of the look-up tables for Raptor decoding is $N \times N_{E}$ bits, where $N_{E}$ is the number of erased symbols, which should be negligible compared to the size of one block.

We have also examined required computation complexity of Raptor decoding in the proposed recovery mode. It is known that decoding complexity of $K$ Raptor symbols requires $O(K)$ symbol additions [8]. In the proposed scheme, there is an additional computation burden to construct the look-up tables, requiring a total of $O(K \sqrt{K})$ binary additions. Since the size of the Raptor symbol in the proposed recovery scheme is in the order of $1 \mathrm{~KB}$, when the number of source symbols is set to 8000 , the additional amount of computation can be roughly estimated as hundreds of symbol additions. This represents an increase in the overall computation by less than $2 \%$ of that required for original Raptor decoding.

\section{Practical Data-Management Issues}

Recall that the proposed scheme is applied to logical blocks. When one logical block is written, data in this block is typically distributed to multiple physical blocks. In this scenario, some problems arise. These problems and their possible solutions are described as follows.

1) While data is written, parity symbols should be updated repeatedly. Thus, before the writing of one block of data is finished, the corresponding parity symbols are maintained in a rewritable memory, which we assume to be static random access memory (SRAM). After one block of data is completely written, the updated parity symbols are then written in the flash memory cells. Note that SRAM is not needed for all physical blocks; the number of parity symbol sets that need be maintained in SRAM is the same as the maximum number of parallel input streams handled by NAND flash.

2) When data writing is suspended for the given block with only a portion of NAND block filled up, the calculated parity symbols can be written to NAND flash memory right away, or they are held in SRAM until the block gets filled up later on. The former method can reduce the use of SRAM, but when the rest of the data are written in the block, the written parity symbols should be updated, which would mean invalidating the pages that contain the existing parity symbols. This would cause more wasted flash memory space as new parity symbols must be written onto new physical pages. The latter approach, on the other hand, can avoid the waste of space, but SRAM is required to house the parity symbol sets for all unfilled blocks, which would not be practically feasible. In reality, a judicious mix of two strategies would be desirable.

3) When some pages of the block are updated, new data must be written to new physical pages and the out of date pages are declared invalid. In this case, to update the parity symbols, the invalid pages and the current parity symbols should be read. In the use of RAIDs, this is also an inevitable problem. To reduce the cost of parity update, methods such as Partial Parity Cache (PPC) [22] and Flash-aware Redundancy Array (FRA) [27] have been proposed, which can also be employed for our purposes here.

In SSDs, the concept of internal parallelism is built-in to increase the bandwidth of reading and writing. In an SSD, flash memory chip packages are connected to the controller through multiple channels, and each flash memory package includes two or more chips. Each chip can be selected individually and perform a read, write or erase operation independently. Hence, when there exist 16 channels and 4 chips per package, for example, the internal parallelism degree will be 64 and the maximum number of the partially filled blocks will be 64 . Thus, in the proposed scheme, to solve the problems described in scenarios 1) and 2), the whole size of the Raptor parity symbol sets that need be maintained in SRAM is 64 times the size of the Raptor parity symbol. This would mean that the required SRAM buffer size is less than $2.6 \mathrm{MB}$, assuming that the block size is $2 \mathrm{MB}$ and the Raptor code has a $2 \%$ coding overhead.

Before ending this subsection, we remark that because the proposed block recovery method actually deals with logical blocks rather than physical blocks, the idea may be utilized for recovery of data stored across multiple NAND chips, somewhat akin to RAID. The potential issue that needs be carefully addressed here, however, is that the size of the blocks needs be large enough to ensure the efficiency of the Raptor code, which may lead to system-level challenges for handling long decoding latency.

\section{B. Performance Evaluation}

Recall that one inner codeword covers $N_{s}$ Raptor symbols exactly, $K$ is the number of inner codewords in a block, and 
the bit size of $N-K$ inner codewords are the same as that for the $N_{s}(N-K)$ parity symbols. The failure rate of the Raptor decoder, given that the number of the failed inner codes $N_{E}$ is $i$ and $N_{s} \cdot i$ erasure symbols are produced, is given by

$$
P\left(E_{1} \mid N_{E}=i\right)=\min \left\{1,2^{-\left(N_{s}(N-K)-N_{s} \cdot i\right)}\right\}
$$

making use of the expression developed in [15]. The R10 code has performance close to that of a random binary fountain code. So, when the receiver collects $C(=S+R)$ symbols except the erased symbols, where $S$ is the number of source symbols to be decoded, the probability of decoding failure of the R10 code is roughly $2^{-R}$, which is also the probability that a $C$ by $S$ random binary matrix is full rank. The min function is used because when $R$ is less than 0 the decoding failure rate is 1 .

When the $(n, k, t) \mathrm{BCH}$ code is used, the erasure probability of the inner code can be obtained by

$$
P_{E}=1-\sum_{i \leq t}\left(\begin{array}{c}
n \\
i
\end{array}\right) p_{e}{ }^{i}\left(1-p_{e}\right)^{n-i}
$$

where $p_{e}$ is the raw bit error rate (BER). Then the error probability $P_{F}$ of the proposed concatenated codes is the probability of the events that decoding fails in the Raptor code (event $E_{1}$ ) or any miss correction occurs over the whole words in the block (event $E_{2}$ ). It can be easily shown that $P_{F}$ satisfies:

$$
\begin{aligned}
& P_{F} \\
& =P\left\{E_{1} \text { or } E_{2}\right\} \approx P\left\{E_{1}\right\}+P\left\{E_{2}\right\} \\
& =\sum_{i=1}^{i=K} P\left(E_{1} \mid N_{E}=i\right) P\left(N_{E}=i\right)+1-\left(1-P_{\text {u.e. }}\right)^{K} \\
& \leq \sum_{i=1}^{i=K}\left[\left(\begin{array}{c}
K \\
i
\end{array}\right) P_{E}{ }^{i}\left(1-P_{E}\right)^{K-i} \min \left\{1,2^{-\left(N_{s}(N-K)-N_{s} \cdot i\right)}\right\}\right] \\
& +K \cdot P_{u e},
\end{aligned}
$$

where $P_{u e}$ is the undetected error (miss correction) probability of the inner ECC. Hence, if the miss-correction probability and the erasure probability of the ECC are known, then the error rate performance can be predicted.

Equation (13) clearly confirms that to achieve a low decoding failure rate for the Raptor code, the miss correction probability of the inner code should be low and the number of parity symbols of Raptor codes should be sufficiently large. To satisfy both conditions with a high code rate, a large enough data size is required. One NAND block contains data in the order of mega bytes, satisfying both conditions when the Raptor code is applied for block recovery.

For the system performance, we have checked the probability of decoding failure in Raptor decoding conditioned on a variable number of uncorrectable pages. We assume that one block consists of 256 pages with each page having $8 \mathrm{~KB}$ of user data corresponding to 8 inner codewords of size $1 \mathrm{~KB}$ each. In this setting, varying $N_{S}$ from 1 to 4 , and the Raptor coding overhead from $1.17 \%$ to $2.34 \%$, we have designed

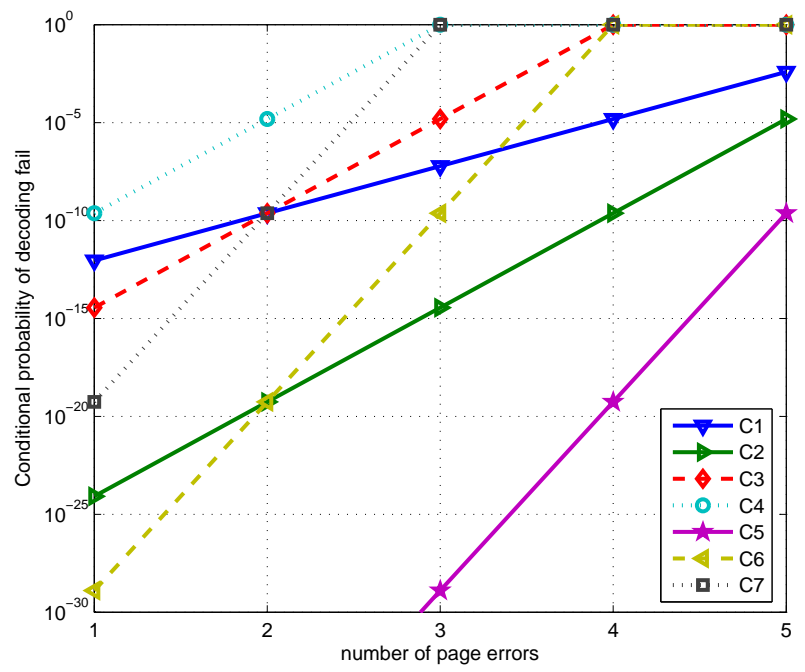

Fig. 3. Conditional probability of Raptor decoding failure given the number of bad pages in a block for the 7 codes specified in Table $\square$ Solid lines are for the codes with a $2.34 \%$ overhead $\left(C_{1}, C_{2}\right.$, and $\left.C_{5}\right)$ while dashed lines correspond to the codes with a $1.56 \%$ overhead $\left(C_{3}, C_{6}\right)$, and dotted lines are for the codes with a $1.17 \%$ overhead $\left(C_{4}, C_{7}\right)$.

seven Raptor codes $\left(C_{1}-C_{7}\right)$ with the parameters given in Table [I With the extra coding overhead defined as the ratio of the number of Raptor parity pages to the number of source pages, the overheads of $2.34 \%, 1.56 \%$ and $1.17 \%$ mean additional 6, 4 and 3 pages for Raptor parity, respectively.

For the chosen code parameter selections, the analytical results for the Raptor decoding failure rate are summarized in Fig. 3 For $C_{1}, C_{2}$ and $C_{5}$, which utilize a $2.34 \%$ overhead, the performance curves are shown in solid lines, and for the codes utilizing a $1.56 \%$ overhead $\left(C_{3}, C_{6}\right)$ performances are represented by dashed lines. Given the same overhead, as $N_{s}$ is increased, the decoding performance improves since the Raptor codes can utilize more parity symbols. For $C_{5}$, which use $N_{s}=4$ and a $2.34 \%$ overhead, the Raptor decoder can recover the block for up to 5 failed pages with conditional decoding failure rates of better than $10^{-10}$. For codes with the same $N_{s}$ but with smaller coding overheads, $C_{6}$, with a $1.56 \%$ overhead, can achieve conditional decoding failure rates

\section{TABLE I}

Different Raptor code parameters with different symbol sizes and numbers of parity symbols. $N_{s}$ represents the number of Raptor symbols within each inner codeword of length $1 \mathrm{~KB}$. SS and PS denote source symbols and parity symbols, respectively. The overhead of $2.34 \%$ means additional 6 pages are used as the parity pages; the overhead of $1.56 \%$ and $1.17 \%$ mean 4 and 3 parity pages, respectively.

\begin{tabular}{c||c|c|c|c|c}
\hline Cases & $N_{s}$ & No. of SSs & Symbol size & No. of PSs & Overhead \\
\hline$C_{1}$ & 1 & 2048 & $1 \mathrm{~KB}$ & 48 & $2.34 \%$ \\
\hline$C_{2}$ & 2 & 4096 & $1 / 2 \mathrm{~KB}$ & 96 & $2.34 \%$ \\
\hline$C_{3}$ & 2 & 4096 & $1 / 2 \mathrm{~KB}$ & 64 & $1.56 \%$ \\
\hline$C_{4}$ & 2 & 4096 & $1 / 2 \mathrm{~KB}$ & 48 & $1.17 \%$ \\
\hline$C_{5}$ & 4 & 8192 & $1 / 4 \mathrm{~KB}$ & 192 & $2.34 \%$ \\
\hline$C_{6}$ & 4 & 8192 & $1 / 4 \mathrm{~KB}$ & 128 & $1.56 \%$ \\
\hline$C_{7}$ & 4 & 8192 & $1 / 4 \mathrm{~KB}$ & 96 & $1.17 \%$ \\
\hline
\end{tabular}


below $10^{-10}$ up to 3 corrupted pages and $C_{7}$, with a $1.17 \%$ overhead, can correct 2 pages with a conditional failure rate of $10^{-10}$.

\section{Raptor Codes with Block-Wise Product Codes}

In this section, the second Raptor-based concatenation scheme designed for page error correction during the normal read mode is discussed. The proposed scheme uses the BWPC of [7] as the inner code to declare erasure for the shared bits among failed row codes and column codes after inner decoding. The overall code structure and the code parameter selection process are described. Performance analysis along with simulation results are provided.

\section{A. Code Structure}

The BW-PC consists of row and column constituent codes that share multiple coded bits in their intersections [7]. In this sense, this code can be viewed as a generalization of the conventional row-column product code where every pair of row and column codes shares one bit. The advantage of the block-sharing is that for a fixed-size two-dimensional array, larger (and thus stronger) row and column codes can be employed. The disadvantage is that when a given pair of row-column codes fail to decode, error locations can only be identified down to the shared block of the failed row and column. The work of [7] has shown that with iterative harddecision decoding, an error floor appears in the mid-to-low BER region due to dominant error events. In [7], this error floor has been lowered significantly by iterative reliabilitybased decoding, with the reliability information acquired based on memory-sensing via multiple reads. In this work, we instead apply an outer Raptor code to lower the error floor without resorting to multiple reads.

For the BW-PC, binary BCH codes are considered as component codes. Let the codeword length, message length and error-correcting capability of the row and column codes be characterized by $\left(n_{r}, k_{r}, t_{r}\right)$ and $\left(n_{c}, k_{c}, t_{c}\right)$, respectively. Let $B_{i, j}$ denote a block of shared bits between the $i$-th row code and $j$-th column code. Let $n_{B}$ be its size in bits, which is a key design parameter. This block of $n_{B}$ bits act as one or more $\left(N_{i}\right)$ Raptor symbols in our setting. See Fig. 4 The symbols $R_{i}^{r}$ and $R_{j}^{c}$ represent the blocks of parity bits for the corresponding row and column binary $\mathrm{BCH}$ codes, respectively. Let $m_{r}$ and $m_{c}$ be the respective parity lengths of the row and the column codes such that $m_{r}=n_{r}-k_{r}$ and $m_{c}=n_{c}-k_{c}$.

When one block is used as one Raptor symbol (i.e. $N_{i}=1$ ), at the outer encoder, for every $K$ source symbols $\left(K n_{B}\right.$ source bits), $N-K$ Raptor parity symbols are first generated as described in Section $\amalg-\mathrm{B}$. The resulting $n_{B}$-bit symbols are then arranged into a $k_{r}^{B}$-row by $k_{c}^{B}$-column array such that $k_{r}^{B}\left(k_{c}^{B}\right)$ is $k_{r} / n_{B}\left(k_{c} / n_{B}\right.$, respectively). When $N_{i}$ is greater than one, the numbers of source symbols and parity symbols of the outer Raptor code increase to $N_{i} N$ and $N_{i}(N-k)$, respectively. Also, each block $B_{i, j}$ contains $N_{i}$ Raptor symbols of $n_{B} / N_{i}$ bits each.

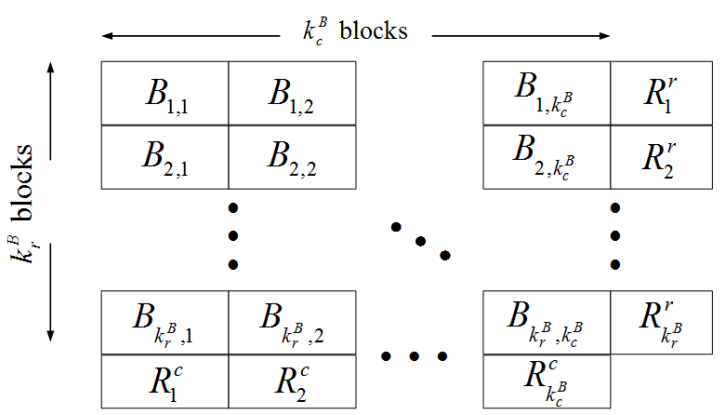

Fig. 4. Structure of BW-PC

For each row of $k_{c}^{B}$ blocks, parity bits for a binary $\mathrm{BCH}$ code are computed taking the corresponding $k_{c}^{B} n_{B}$ bits as the message bits, and these parity bits form the symbols $R_{i}^{r}$ in Fig. 4 Binary BCH codes for the columns are also constructed in the same fashion. The overall code rate is the product of two code rates $\frac{k}{n} \frac{K}{N}$, where $k$ is the message length of the inner BW-PC which is equal to $k_{r}^{B} k_{c}^{B} n_{B}$ and $n$ is the corresponding codeword length which is given by $k_{r}^{B} k_{c}^{B} n_{B}+k_{r}^{B} m_{r}+k_{c}^{B} m_{c}$.

For decoding, the component row-column $\mathrm{BCH}$ decoders perform iterative hard-decision decoding first. Each row component code is decoded via normal hard-decision $\mathrm{BCH}$ decoding (e.g., Berlekamp-Massey decoding). The columns decoders are then executed taking the hard decisions generated by the row decoders, completing one round of hard-decision iteration 2 . Iteration continues until all the errors are corrected, or an additional round does not correct the remaining errors. In the latter case, decoding failure is declared and erasure tags are applied to all symbols that reside in the intersecting blocks of the failed row and column codes. Raptor decoding is then executed as explained in $\amalg-\mathrm{B}$

\section{B. Code Design}

We assume a page size of $8 \mathrm{KBs}$ and the target page error rate (PER) is set below $10^{-12}$. The overall code rate is set to 0.93 . Given the user data of $k=2^{16}$ bits, we set out to choose reasonable values for the six parameters: $n_{B}, k_{r}, k_{c}, t_{r}, t_{c}$ and $N$.

First, recall that for the Raptor code the decoding error probability depends on the number of parity symbols. Given the fairly high-code-rate requirement of practical NAND flash memories, to realize the full performance potential of Raptor codes, a large number of Raptor symbols are necessary per Raptor codeword. We shall only consider Raptor codes with at least 1000 symbols. This sets the limit on the size of the Raptor symbol: namely, $n_{B}=k / N$ should be less than or equal to 64. After the value for $n_{B}$ is chosen, $k_{r}$ and $k_{c}$ as well as $t_{r}$ and $t_{c}$ are chosen to provide strong enough inner ECC protection while satisfying the overall code rate requirement of 0.93 with a comfortable margin. Finally, $K$ is chosen that ensures the final target PER while meeting the overall code rate $R$ of 0.93 .

\footnotetext{
${ }^{2}$ Variations are possible on iteration scheduling as well as on the implementation of hard decision feedback between the row-column decoders.
} 
Of the various codes that have been constructed and examined, we include two representative cases in Table II Each intersection contains $N_{i}$ Raptor symbols. Increasing $N_{i}$ ensures reliable Raptor decoding performance, but the number of the source symbols $\left(N_{i} K\right)$ should also not exceed 8192, the source size of the employed R10 code. $C_{1}$ represents the combination of a weak inner code with a strong outer code while $C_{2}$ exemplifies that of a strong inner code with a weak outer code.

\section{Performance Analysis and Evaluation}

The analysis of decoding failure can be carried out by obtaining the probability distribution of the number of erased symbols in hard-decision iteration decoding of BW-PCs. We make use of the analysis given in [7] for the BW-PC failure rate in arriving at an expression for the final Raptor decoder failure rate.

To briefly summarize the analysis of [7], let $\epsilon_{i, j}$ be the error event corresponding to the failure of $i$ rows and $j$ columns and let $P_{i, j}$ denote the corresponding error event probability. Then, the decoding failure probability $P_{F}$ of the inner BW-PC can be expressed as follows:

$$
P_{F}=\sum_{(i, j)} P_{i, j}
$$

with

$$
\begin{aligned}
P_{i, j}= & \left(\begin{array}{c}
k_{r}^{B} \\
i
\end{array}\right)\left(\begin{array}{c}
k_{c}^{B} \\
j
\end{array}\right) \sum_{n_{1}=0}^{n_{B}} \sum_{n_{2}=0}^{n_{B}} \cdots \sum_{n_{i \times j}=0}^{n_{B}} \sum_{n_{\alpha_{1}}=0}^{m_{r}} \cdots \sum_{n_{\alpha_{i}}=0}^{m_{r}} \\
& \sum_{n_{\beta_{1}}=0}^{m_{c}} \cdots \sum_{n_{\beta_{j}}=0}^{m_{c}}\left(\begin{array}{c}
n_{B} \\
n_{1}
\end{array}\right)\left(\begin{array}{c}
n_{B} \\
n_{2}
\end{array}\right) \cdots\left(\begin{array}{c}
n_{B} \\
n_{i \times j}
\end{array}\right) \\
& \left(\begin{array}{c}
m_{r} \\
n_{\alpha_{1}}
\end{array}\right) \cdots\left(\begin{array}{c}
m_{r} \\
n_{\alpha_{i}}
\end{array}\right)\left(\begin{array}{c}
m_{c} \\
n_{\beta_{1}}
\end{array}\right) \cdots\left(\begin{array}{c}
m_{c} \\
n_{\beta_{j}}
\end{array}\right) \\
& P_{e}^{n^{e}}\left(1-P_{e}\right)^{n_{B} \cdot i \cdot j+i \cdot m_{r}+j \cdot m_{c}-n^{e}} \cdot 1_{S}
\end{aligned}
$$

where $P_{e}$ is the average raw $\mathrm{BER} ; n_{1}, n_{2}, \ldots, n_{i \times j}$ are the numbers of errors in the individual intersections of the failed row and column codes; $n_{\alpha_{l}}$ is the number of errors in the parity block $R_{\alpha_{l}}^{r}$ in the failed row codes $(l=1,2, \ldots, i) ; n_{\beta_{p}}$ is the number of errors in the parity block $R_{\beta_{p}}^{c}$ in the failed column code $(p=1,2, \ldots, j) ; n^{e}$ is the total number of errors in the $i \times j$ intersections plus $i$ row parity blocks and $j$ column parity blocks, i.e., $n^{e}=\sum_{k=1}^{i \times j} n_{k}+\sum_{l=1}^{i} n_{\alpha_{l}}+\sum_{p=1}^{j} n_{\beta_{p}}$; $1_{A}$ is the indicator function which is ' 1 ' if and only if the event A is true; and $S$ represents the condition that everyone

\section{TABLE II}

The parameters of Raptor code/BW-PC combinations: the row and column codes are $(n, k, t) \mathrm{BCH}$ codes and $N_{r}$ denotes the number of parity blocks for Raptor codes $\left(N_{r}=N-K\right)$ and the number of parity symbols of Raptor codes is $N_{i} \times N_{r}$.

\begin{tabular}{c||c|c|c|c|c}
\hline Overall codes & $n_{B}$ & $N_{i}$ & Row code & Column code & $N_{r}$ \\
\hline \hline$C_{1}$ & 14 & 1 & $(996,966,3)$ & $(996,966,3)$ & 58 \\
\hline$C_{2}$ & 56 & 4 & $(2026,1960,6)$ & $(1970,1904,6)$ & 7 \\
\hline
\end{tabular}

of the $i \cdot j$ particular selected row and column codes fail to decode.

The probability of the most dominant event $\epsilon_{1,1}$, for example, is given by

$$
\begin{aligned}
P_{1,1}= & k_{r}^{B} \times k_{c}^{B} \sum_{n_{1}^{e}=0}^{n_{B}} \sum_{n_{2}^{e}=0}^{m_{r}} \sum_{n_{3}^{e}=0}^{m_{c}}\left(\begin{array}{c}
n_{B} \\
n_{1}
\end{array}\right)\left(\begin{array}{c}
m_{r} \\
n_{2}
\end{array}\right)\left(\begin{array}{c}
m_{c} \\
n_{3}
\end{array}\right) \\
& P_{e}^{n^{e}}\left(1-P_{e}\right)^{n_{B}+m_{r}+m_{c}-n^{e}} \cdot 1_{S} .
\end{aligned}
$$

where $n_{1}^{e}, n_{2}^{e}$, and $n_{3}^{e}$ are the number of errors in message block $B_{i, j}$ intersected by a pair of failed row and column codes, the number of errors in the parity block $R_{i}^{r}$ in the failed row code, and the number of errors in the parity block $R_{j}^{c}$ in the failed column code, respectively. Also, $n^{e}=n_{1}^{e}+n_{2}^{e}+n_{3}^{e}$ and $S$ is the condition for the decoding failure event : 1) $t_{r}<$ $n_{1}^{e}+n_{2}^{e}$ and 2) $t_{c}<n_{1}^{e}+n_{3}^{e}$.

In this paper, we have evaluated $P_{i, j}$ for $(i, j)=$ $(1,1),(1,2),(2,1),(2,2),(3,1),(1,3)$. Adding more terms did not increase the overall decoding failure rate for the $\mathrm{BW}$ PC. Now, the failure rate of Raptor decoding, denoted by $P_{\text {Raptor }}$, can be obtained as

$$
\begin{aligned}
P_{\text {Raptor }} & =\sum_{l=1}^{l=N} P\left(E_{1} \mid N_{E}=l\right) P\left(N_{E}=l\right) \\
& =\sum_{i=1}^{k_{r}^{B}} \sum_{j=1}^{k_{c}^{B}}\left[\min \left\{1,2^{-N_{i}(N-K-i \cdot j)}\right\} \cdot P_{i, j}\right]
\end{aligned}
$$

where $N_{E}$ denotes the number of erasure blocks, which is equal to the total number of intersections among the failed columns and rows.

The results of the analysis as well as simulations are summarized in Figs. 5 and 6 , In each figure, the curve labeled by 'Sim' represents the simulated PERs right after harddecision iteration decoding is done for the inner BW-PC. The curves labeled ' $P_{i, j}$ ' are the results of evaluating (15) for the corresponding error events. Finally, the curve labeled by 'P $P_{\text {Raptor' }}$ is the analysis result corresponding to 17. It can be seen that in each figure, the simulation results for the BW-PC decoding closely follows $P_{1,1}$ in the floor region, indicating that the performance is dominated by the $\epsilon_{1,1}$ event.

Generally speaking, when the inner BW-PC gets strong (as characterized by long codewords and/or low code rates), the probability of dominant error events tend to stay low and the PER curve drops quickly to the error floor. The overall performance indicated by $P_{\text {Raptor }}$, however, also depends strongly on the strength of the Raptor code (which improves with increasing number of redundant Raptor symbols $N_{i} N_{s}$ and/or decreasing size of the symbol $n_{B}$ ).

For example, $C_{1}$ in Fig. 5 consists of a weak inner BW-PC code and a strong outer Raptor code. In comparison with $C_{2}$ of Fig. 5, which combines a strong inner code with a weak outer code, it can be seen that the $P_{i, j}$ curves for $C_{1}$ are substantially higher, while the overall performance curve $P_{\text {Raptor }}$ appears considerably better with $C_{1}$. This is due to the powerful outer code of $C_{1}$ correcting the dominant errors that remain after inner decoding.

Would $C_{1}$ then be a reasonable code, given its superior $P_{\text {Raptor }}$ curve that stays well below the target PER at all raw 


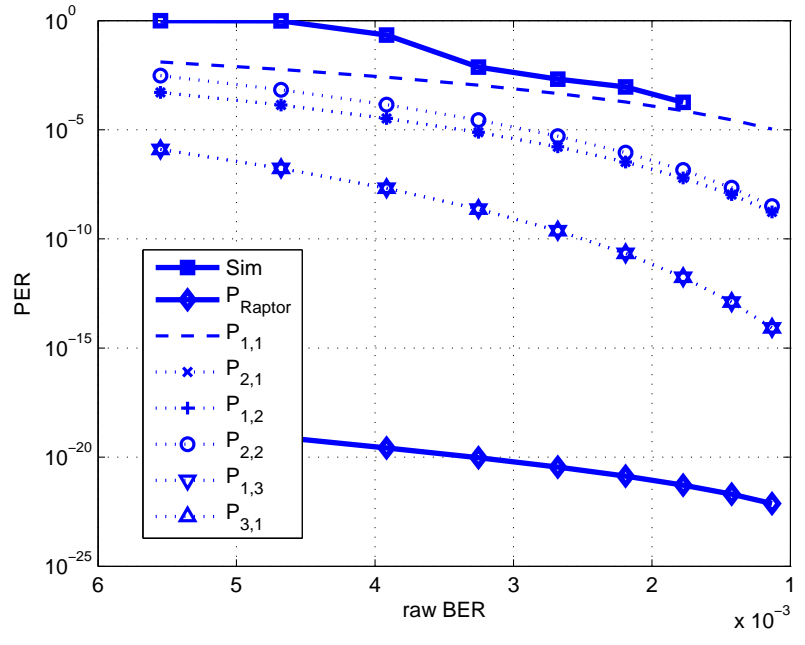

Fig. 5. PER simulation and dominant error probability of Raptor codes with BW-PC $C_{1}$

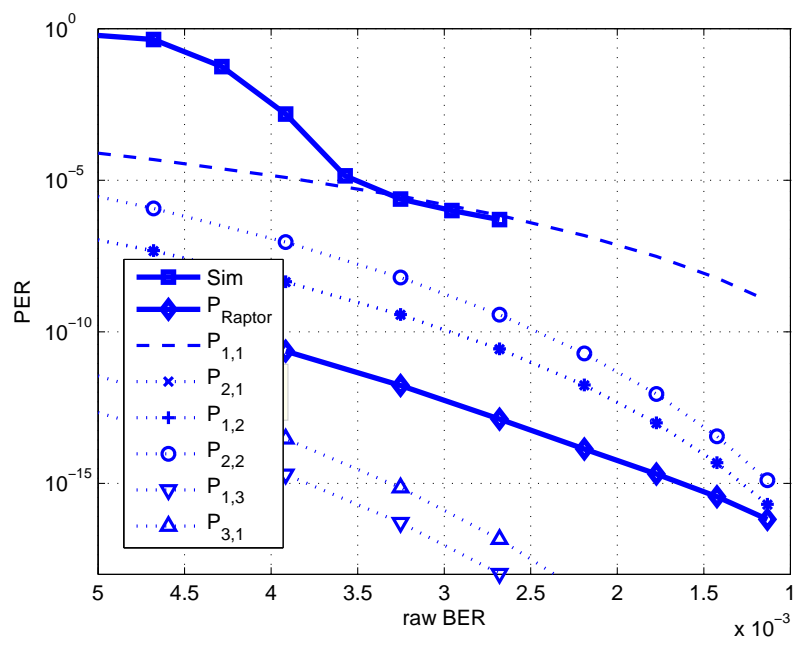

Fig. 6. PER simulation and dominant error probability of Raptor codes with BW-PC $C_{2}$

BER values as seen in Fig. 5? Unfortunately, the answer turns out to be negative, since there were too many miss-correction errors (i.e., decoding to a valid but incorrect codeword) observed after inner decoding is done, implying that in this case the analysis predicted by (17) is not reliable. This is due to the fact that in $C_{1}$, the weak component codes are prone to miss-correction.

The finding that the analytical tool established by 17 may be unreliable in certain cases obviously raises a serious concern regarding the credibility of the low error rate analysis of 177. In hopes of getting around this issue, for various inner code parameters, we checked via simulation for the presence of any miss-correction events in all row and column codes, using relative weak component codes for which Monte-Carlo simulation is feasible with reasonable computer time. Here, we could observe two important trends:

1) After hard-decision-iterative decoding is done, the miss-

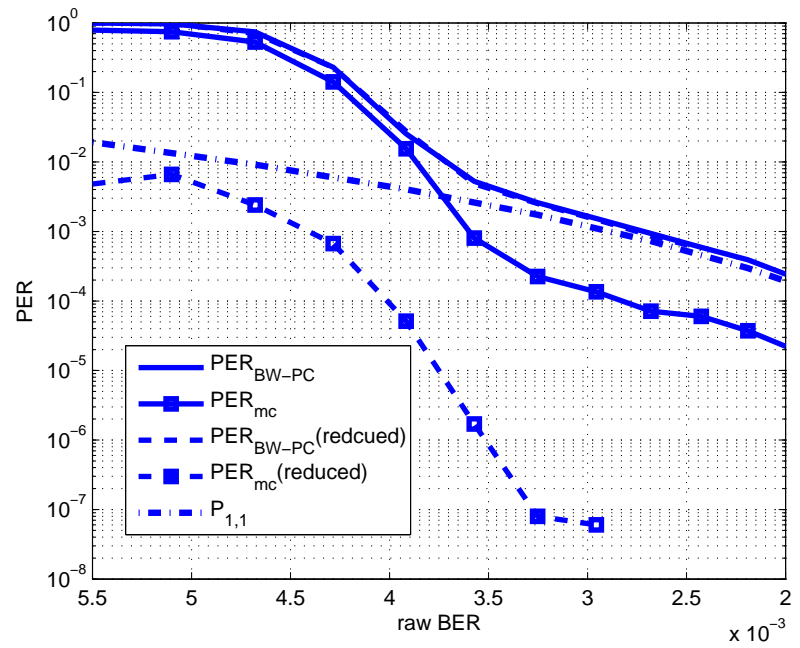

Fig. 7. Miss-correction probability $\left(\mathrm{PER}_{m c}\right)$ and decoding failure probability $\left(\mathrm{PER}_{\mathrm{BW}-\mathbf{P C}}\right)$ of an example BW-PC code with weak components codes with and without decoding sphere reduction (from $t=3$ to 2 for each component code). $\mathrm{P}_{F}$ is the bound of decoding failure probability of BW-PC obtained in (14).

correction probability curve forms a floor due to the occurrences of some dominant events, as the raw BER is allowed to improve. Specifically, as the channel noise drops and thus the corresponding raw BER reduces down to a particular value, dominant miss-correction events appear as errors detected by the first component code but not by the second. Let us call these events half detected errors.

2) The onset of the floor in the miss-correction rate curve occurs at the same raw BER value as the onset of the floor for the simulated page error rate for the iterative inner decoding. Note that the onset of the floor in the PER curve marks the occurrence of the dominant $\epsilon_{i, j}$ events. In addition, the onset of the floor in the miss-correction rate corresponding to a reduced decoding sphere (the motivation for which is to be given shortly) also coincides with that of the PER.

Fig. 7 shows the simulated decoding failure rates or the PERs after inner iterative decoding as well as the simulated rates of the miss-correction events (denoted by $\mathrm{PER}_{m c}$ ) of an example BW-PC code with relatively weak components codes given by $(1057,1024,3)$ and $(1057,1024,3)$ with $n_{B}=16$. We observed that both the decoding failure rate and the misscorrection rate start to get dominated by respective particular events as the raw BER reduces down to $3.3 \times 10^{-3}$. Also seen in Fig. 7 is the PER $_{m c}$ corresponding to a case where the decoding sphere is reduced from $t=3$ to $t=2$. The PER corresponding to the reduced decoding sphere is increased only slightly (thus nearly indistinguishable in the figure) relative to the PER corresponding to the original decoding sphere set to $t=3$. On the other hand, it can be seen that the miss-correction rate for the reduced decoding sphere is improved dramatically.

A reduced decoding sphere provides a means to alleviate the miss-correction issue during inner code decoding. If a half detected error event is indicated after the inner BW-PC 
decoding is completed, a simple strategy is to go back to the inner decoding stage and run the decoders again with a reduced decoding sphere. As the simulation results indicate, this will typically decrease the miss-correction rate by a substantial amount while affecting the decoding failure rate of the overall code very little.

The trends about the onset of the floor and the dominance of particular events have been observed consistently over various BW-PC codes with relatively weak component codes. If this trend is true for the stronger component codes used in $C_{2}$, for example, then we can safely conclude that the miss-correction rate of the inner code is suppressed to a negligible level using reduced-sphere inner decoding. In Fig. 8, simulation results for $C_{2}$ 's decoding failure probability (PER) and inner misscorrection probability $\left(\mathrm{PER}_{m c}\right)$ with and without decoding sphere reduction (from $t=6$ to 5 ) are summarized. The dotted portions of the PER $_{m c}$ curves represent extrapolation under the assumption that the onset of the floor does not appear until the PER curve is dominated by the $\epsilon_{i, j}$ events. This onset is observed at the raw BER of $3.3 \times 10^{-3}$, as indicated by a vertical line segment. As the page miss-correction rate reaches below $10^{-12}$, the overall target page error rate, we can start to ignore miss-correction events at the inner decoding and the overall code performance will be accurately estimated by 177 .

We have already seen that $P_{\text {Raptor }}$ of $C_{2}$ in Fig. 5 roughly meets the target PER of $10^{-12}$ at the raw BER of $3.3 \times 10^{-3}$. This, combined with the conjecture made in Fig. 8 that the miss-correction rate at inner decoding also falls below $10^{-12}$, indicates that the $C_{2}$ code achieves an acceptable page error rate for raw BERs as high as $3.3 \times 10^{-3}$. We again remark that this conjecture is based on observing that for relatively weak components codes of various BW-PC inner codes that allow decoding simulation with reasonable computer time, the onset of the floor for the miss-correction rate curves for the original and reduced decoding spheres coincide with that for the decoding failure rate curve for the hard-decision iterative inner decoding.

We finally note that when the page size increases and thus even stronger inner component codes can be employed, the problem associated with miss-correction at inner decoding will naturally disappear. The current trend in NAND flash system design is to employ increasingly large page formats which tends to boost system throughput in many applications including mobile devices. The proposed concatenation scheme would provide an even more attractive ECC option in these applications.

\section{Performance Comparison with Other Codes}

To compare PER performance of the proposed system with the conventional ECC schemes, we consider the $\mathrm{BCH}$ codes of various lengths as well as an LDPC code of length $1 \mathrm{~KB}$. The results are summarized in Fig. 9 For BCH codes with lengths $1 \mathrm{~KB}-8 \mathrm{~KB}$, the corresponding word error rates have been computed and then weighted by factors $8-1$, respectively, to account for the different codeword sizes in evaluating the PER. The codes are specifically $(8808,8192,44),(17614,16384$, $82),(35232,32768,154)$ and $(70534,65536,294) \mathrm{BCH}$ codes,

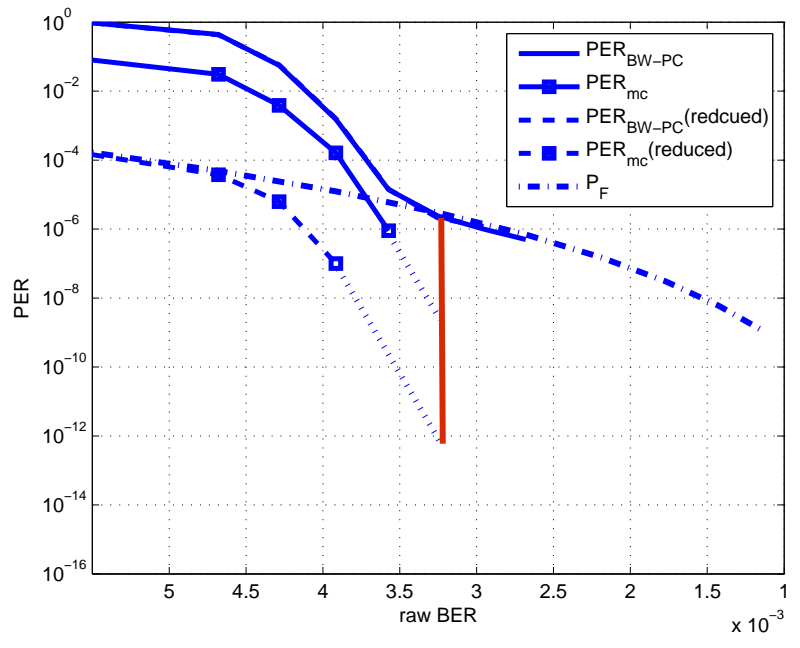

Fig. 8. Miss-correction probability $\left(\mathrm{PER}_{m c}\right)$ and decoding failure probability (PER) of the $C_{2}$ code with and without decoding sphere reduction (from $t=6$ to 5 for each component code). The dotted lines are the low-rate extrapolation for PER $\mathrm{P}_{m c}$ based on the assumption that the onset of the error floor coincide between PER and $\mathrm{PER}_{m c}$.

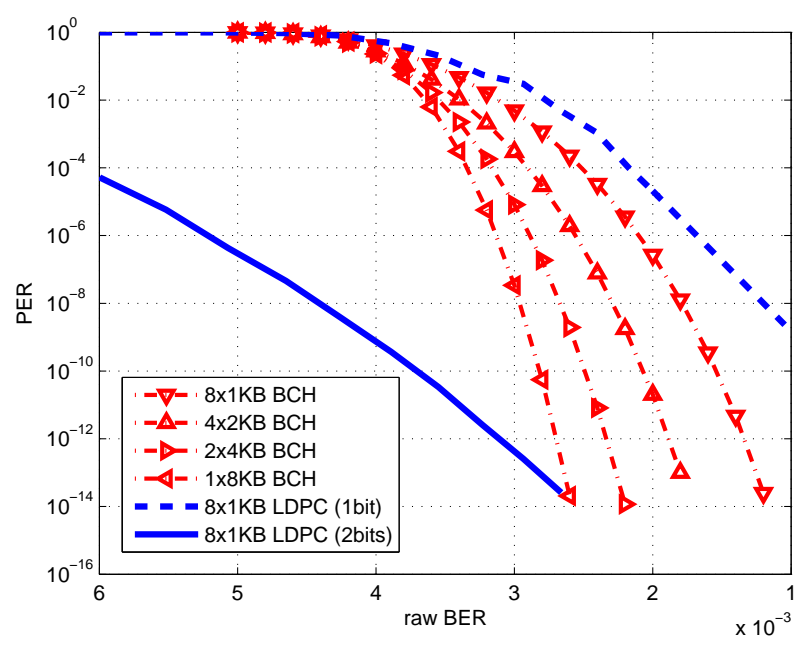

Fig. 9. PERs for long BCH codes with different codeword sizes and LDPC codes with limited-precision sensing. PERs of LDPC codes in the high-rate region are evaluated by Monte-Carlo simulation using $1 \mathrm{~KB}$ LDPC codes and correspond to the probability that any of the $8 \mathrm{LDPC}$ codes in a given page fails. Linear extrapolation (in log-log scale) is used to obtain the PERs below $10^{-6}$.

all corresponding to a code rate of roughly 0.93 . Also included are the simulation results for a quasi-cyclic (QC) LDPC code [28]-[30] with a length of 8249 bits and a variable node degree of 5 , a check node degree of 73 and a sub-array size of 113. The progressive-edge-growth (PEG) algorithm of [31] is used to generate the particular parity check matrix. The LDPC decoder uses the scaled min-sum algorithm [32], [33] based on 1-bit and 2-bit input quantization. The quantization levels are found based on maximization of mutual information [5]. Full internal log-likelihood ratio (LLR) quantization is used for decoding with a maximum of 50 iterations.

By comparing the raw BER values the given codes can 
tolerate in achieving the $10^{-12}$ PER, it can be seen that the proposed scheme gives superior performance relative to even the most powerful $\mathrm{BCH}$ code of length $8 \mathrm{~KB}$. Compared to the LDPC code (the low rate performance of which has been based on extrapolation assuming no error floor exists), the proposed scheme's performance is comparable to that of LDPC code with 2 bit input-quantization, which would require 3 reads (3 different sensing levels). For higher level quantization, the LDPC code will obviously outperform the propose scheme that relies only on hard-decision sensing. As is widely known, the LDPC code with just 1-bit quantization performs quite poorly.

From the complexity standpoint, it is well known that the decoding complexity of $\mathrm{BCH}$ codes is roughly $O(n t)$ [34]. Even after considering that the proposed scheme requires iteration ( $2 \sim 3$ times on the average), the complexity advantage of our scheme over stand-alone $\mathrm{BCH}$ codes becomes obvious mainly due to the much smaller component code sizes. Compared to the LDPC code, again, the performance/complexity tradeoff advantages are clear.

As noted earlier, the RS codes are another clear option for the outer erasure code. For example, with a 10-bit symbol [with symbols taken from $G F\left(2^{10}\right)$ ], seven RS codes can be used to cover one $8 \mathrm{~KB}$ page. In this case, the inner BW-PC can be constructed to collect 70 bits or seven RS symbols in each column-row intersection. With proper interleaving, all seven symbols in a given intersection will spread out to seven different RS codewords. Overall, the RS codes in this example can correct eight erasures from the inner BW-PC. While more refined error rate performance comparison will depend on detailed inner BW-PC parameter optimization, it is fair to say that this particular RS code configuration yields results comparable to the Raptor code designs presented in this paper.

In general, however, the decoding complexity of RS codes is considerably higher than that of Raptor codes. It is well known that decoding an $\mathrm{RS}$ code in $G F(q)$ requires $O\left[q(\log q)^{2}\right]$ symbol operations [35]; in the above scenario RS decoding requires $O\left(10^{5}\right)$ symbol operations (multiplication and addition). On the other hand, although hardware implementation options have not been explored extensively in the literature thus far, it is safe to say that Raptor decoding could be done with linear-time complexity of $O(K)$, as seen in the case of the R10 code [15]. In particular, in the proposed setting discussed in this paper, Raptor decoding requires $O\left(10^{3}\right)$ symbol operations (exclusive-ORing). It is also worth stressing again that there is a definite trend toward increased page sizes for flash memories to improve system throughput [36], [37]. With larger page sizes, the performance/complexity tradeoff advantage of Raptor codes over RS codes will become more significant.

\section{CONClusion}

This paper has considered application of fixed-rate Raptor codes to NAND flash memory. Both an off-line block recovery mode and a real-time page error correction mode have been discussed. Taking advantage of the linear-time encoding/decoding capability of very long Raptor codes, significant performance potentials for the BCH-Raptor concatenation have been demonstrated. The suggested schemes both assume hard-decision sensing of memory cells, and the inner code's decoder convert the hard-decision input/output storage channel into a packet-erasure channel for the outer Raptor decoder. An efficient table-look-up-based encoding/decoding algorithm has been suggested for block recovery that takes advantage of the unique feature of the storage channel, namely, that the data is stored in the channel medium for the receiver to access it at any time. The suggested page-error-correction strategy, on the other hand, utilizes symbol-wise concatenation of relatively small $\mathrm{BCH}$ codes as the inner code, with the intersections of failed row and column codes tagged as erasure symbols for the outer Raptor code. Accordingly, the number of shared bits at the intersection of the row-column codes becomes a key design parameter for the overall concatenation. The error rate analysis combined with simulation demonstrate interesting new possibilities for error control in NAND flash.

\section{REFERENCES}

[1] K. M. J. Lofgren, R. D. Norman, G. B. Thelin, and A. Gupta, "Wear leveling techniques for flash eeprom systems," U.S. Patent 6850443 , May 2, 2003.

[2] R. G. Gallager, "Low-density parity-check codes," MIT Press, 1963.

[3] Y. Maeda and H. Kaneko, "Error control coding for multilevel cell flash memories using nonbinary low-density parity-check codes," in Proc. 24th IEEE Int. Symp. Defect and Fault Tolerance in VLSI Systems (DFT), Oct. 2009 , pp. $367-375$.

[4] G. Dong, N. Xie, and T. Zhang, "On the use of soft-decision errorcorrection codes in nand flash memory," IEEE Trans. Circuits Syst. I, vol. 58, no. 2, pp. $429-439$, Feb. 2011.

[5] J. Wang, T. Courtade, H. Shankar, and R. Wesel, "Soft information for ldpc decoding in flash: Mutual-information optimized quantization," in Proc. IEEE GlobeCom, Dec. 2011, pp. 1 -6.

[6] C. Berrou, A. Glavieux, and P. Thitimajshima, "Near shannon limit error-correcting coding and decoding: Turbo-codes," in Proc. Int. Conf. Communication, vol. 2, Geneve, Swtzerland, May 1993, pp. 1064-1070.

[7] S. gun Cho and J. Ha, "Concatenated bch codes for nand flash memories," in Proc. IEEE Int. Conf. Communications (ICC), Ottawa, Canada, 2012, pp. 2639-2644.

[8] A. Shokrollahi, "Raptor Codes," IEEE Trans. Inf. Theory, vol. 52, pp. 2551 - 2567, Jun 2006.

[9] J. Byers, M. Luby, M. Mitzenmacher, and A. Rege, "A digital fountain approach to reliable distribution of bulk data," in Proc. ACM SIGCOMM, Vancouver, Canada, Sep 1998, pp. 56-57.

[10] J. Byers, M. Luby, and M. Mitzenmacher., "A digital fountain approach to asynchronous reliable multicast." IEEE J. Sel. Areas Commun., vol. 20, no. 8, Oct. 2002.

[11] A. Venkiah, C. Poulliat, and D. Declercq, "Analysis and design of raptor codes for joint decoding using information content evolution," in Proc. IEEE Int. Symp. Information Theory (ISIT), 2007, pp. 421-425.

[12] S. Kim, S. Lee, and S.-Y. Chung, "An efficient algorithm for ML decoding of raptor codes over the binary erasure channel," IEEE Commun. Lett., vol. 12, no. 8, pp. $578-580$, aug. 2008.

[13] T. Mladenov, S. Nooshabadi, and K. Kim, "Implementation and evaluation of raptor codes on embedded systems," IEEE Trans. Comput., vol. 60 , no. 12 , pp. $1678-1691,2011$.

[14] K. Mahdaviani, M. Ardakani, and C. Tellambura, "On raptor code design for inactivation decoding," IEEE Trans. Commun., vol. 60, no. 9, pp. 2377-2381, 2012.

[15] A. Shokrollahi and M. Luby, "Raptor Codes," Foundations and Trends in Communications and Information Theory, vol. 6, pp. 213-322, 2011.

[16] M. Luby, A. Shokrollahi, M. Watson, and T. Stockhammer, Raptor forward error correction scheme for object delivery, Internet Engineering Task Force, RFC 5053, Sep. 2007. [Online]. Available: http://tools.ietf.org/html/rfc5053

[17] 3GPP TS 25.346 v7.0.0, Technical Specification Group Radio Access Networs; Introduction of the Multimedai Broadcas/Multicast Services (MBMS) in the Radio Access Networks, Mar. 2006. 
[18] ETSI TS 102472 v1.2.1, IP Datacast over DVB-H: Content Delivery Protocols,, Mar. 2006. [Online]. Available: http://www.dvb-h.org

[19] O. Etesami and A. Shokrollahi, "Raptor codes on binary memoryless symmetric channels," IEEE Trans. Inf. Theory, vol. 52, no. 5, pp. 2033 - 2051, May 2006.

[20] Z. Cheng, J. Castura, and Y. Mao, "On the design of raptor codes for binary-input gaussian channels," in Proc. IEEE Int. Symp. Information Theory, june 2007, pp. $426-430$.

[21] M. Balakrishnan, A. Kadav, V. Prabhakaran, and D. Malkhi, "Differential raid: Rethinking raid for ssd reliability," ACM Trans. Storage, vol. 6, no. 2, Jul. 2010.

[22] S. Im and D. Shin, "Flash-aware raid techniques for dependable and high-performance flash memory ssd," IEEE Trans. Comput., vol. 60, no. 1, pp. 80-92, 2011.

[23] M. Luby, "LT codes," in Proc. The 43rd Annual IEEE Symp. Foundations of Computer Science (FOCS), 2002, pp. 271 - 280.

[24] M. Luby, M. Mitzenmacher, M. Shokrollahi, and D. Spielman, "Efficient erasure correcting codes," IEEE Trans. Inf. Theory, vol. 47, no. 2, pp. 569-584, 2001.

[25] M. G. Luby, M. Mitzenmacher, M. A. Shokrollahi, D. A. Spielman, and V. Stemann, "Practical loss-resilient codes," in Proc. 29th annual ACM symp. Theory of computing. New York, NY, USA: ACM, 1997, pp. $150-159$.

[26] D. J. C. MacKay, "Fountain codes," Communications, IEE Proceedings-, vol. 152, no. 6, pp. 1062-1068, 2005.

[27] Y. Lee, S. Jung, and Y. H. Song, "Fra: a flash-aware redundancy array of flash storage devices," in Proc. 7th IEEE/ACM Int. Conf. Hardware/software codesign and system synthesis, 2009, pp. 163-172.

[28] M. Fossorier, "Quasicyclic low-density parity-check codes from circulant permutation matrices," IEEE Trans. Inf. Theory, vol. 50, no. 8, pp. 1788-1793, 2004.

[29] L. Chen, J. Xu, I. Djurdjevic, and S. Lin, "Near-shannon-limit quasicyclic low-density parity-check codes," IEEE Trans. Commun., vol. 52, no. 7, pp. 1038-1042, 2004.

[30] J. L. Fan, "Array codes as low-density parity-check codes," in Proc. 2nd Int. Symp. Turbo Codes, Brest, France,, Sept. 2000, pp. 545-546.

[31] X.-Y. Hu, E. Eleftheriou, and D.-M. Arnold, "Progressive edge-growth tanner graphs," in Proc. IEEE GlobeCom, vol. 2, 2001, pp. 995-1001 vol.2.

[32] J. Chen, R. Tanner, C. Jones, and Y. Li, "Improved min-sum decoding algorithms for irregular ldpc codes," in Proc. IEEE Int. Symp. Information Theory, 2005, pp. 449-453.

[33] J. Zhao, F. Zarkeshvari, and A. Banihashemi, "On implementation of min-sum algorithm and its modifications for decoding low-density parity-check (ldpc) codes," IEEE Trans. Commun., vol. 53, no. 4, pp. 549-554, 2005.

[34] J. Hong and M. Vetterli, "Simple algorithms for bch decoding," IEEE Trans. Commun., vol. 43, no. 8, pp. 2324-2333, 1995.

[35] J. Justesen, "On the complexity of decoding reed-solomon codes (corresp.)," IEEE Trans. Inf. Theory, vol. 22, no. 2, pp. 237-238, 1976.

[36] M. Abraham, "Nand flash architecture and specification trends," Flash Memory Summit, 2012.

[37] H. Vuong, "Flash storage trends and ecosystem," JEDEC Flash Storage Summits, 2010. 